\title{
Reprodutibilidade intra-observador de medidas morfométricas do segmento anterior em olhos normais pela biomicroscopia ultra-sônica (UBM)
}

\author{
Intraobserver reproducibility in anterior segment morphometryof normal eyes using \\ ultrasound biomicroscopy(UBM)
}

\author{
Edmar C. de Souza Filho' \\ Flávio de A. Marig'o ${ }^{2}$ \\ Cristiano Oliveira ${ }^{3}$ \\ Sebastião Cronemberger ${ }^{4}$ \\ Nassim Calixto ${ }^{5}$
}

Trabalho realizado no Serviço de Glaucoma do Hospital São Geraldo, Hospital das Clínicas da Universidade Federal de Minas Gerais - UFMG.

Trabalho baseado em Tese de Doutorado defendida e aprovada na Universidade Federal de Minas Gerais (UFMG). Orientador: Dr. Flávio A. Marigo.

${ }^{1}$ Doutor em Medicina pela Faculdade de Medicina da Universidade Federal de Minas Gerais - UFMG. Belo Horizonte (MG).

${ }^{2}$ Doutor em Medicina pela Faculdade de Medicina da UFMG. Pós-Doutorado em Oftalmologia, New York Medical College, The New York Eye \& Ear Infirmary, NY, USA. Professor convidado do Curso de Pós-Graduação em Oftalmologia (Nível Doutorado) da Faculdade de Medicina da UFMG. Belo Horizonte (MG).

${ }^{3}$ Fellow do Serviço de Glaucoma do Hospital São Geraldo,

Hospital das Clínicas da UFMG. Belo Horizonte (MG).

${ }^{4}$ Professor Titular de Oftalmologia da Faculdade de Medicina da UFMG. Belo Horizonte (MG).

${ }^{5}$ Professor Titular de Oftalmologia da Faculdade de Medicina da UFMG. Belo Horizonte (MG).

Fonte de Financiamento: Bolsa de Doutorado da CAPES.

Endereço para correspondência: Flávio A. Marigo Instituto da Visão. Rua dos Otoni, 881 - 13ํandar

Belo Horizonte (MG) CEP 30150-270

E-mail:fmarigo@terra.com.br

Recebido para publicação em 11.07.2003

Versão revisada recebida em 26.11.2004

Aprovação em 15.12.2004

Nota Editorial: Após concluída a análise do artigo sob sigilo editorial e com a anuência da Dra. Norma Allemann sobre a divulgação de seu nome como revisora dele, agradecemos sua participação nesse processo.

\begin{tabular}{|l|}
\hline \multicolumn{1}{|c|}{ RESUMO } \\
\hline Objetivo: Estudar as variáveis que caracterizama morfometria do segmento \\
anterior usando a biomicroscopia ultra-sônica (UBM), em pacientes com o \\
exame oftalmológico normal, em amostra de tamanho significativo, de forma \\
sistematizada, com o intuito de avaliar sua reprodutibilidade intra-obser- \\
vador. Dois novos parâmetros tiveram sua reprodutibilidade intra-ob- \\
servador também testada. Métodos: Foram examinados 190 olhos de 101 \\
pacientes comexameoftalmológiconormal empregando-seaUBM. Emcada \\
olho, além da profundidade da câmara anterior (PCA), onze outros parâ- \\
metros que caracterizama morfometria do segmento anterior foram medidos \\
nos meridianos superior, nasal, inferior e temporal, em dois momentos \\
distintos com intervalo mínimo de quatro semanas entre eles, pelo mesmo \\
observador. Resultados: Não se observaram diferenças estatisticamente \\
significativas entre a primeira e a segunda medida (M1 e M2) de cada \\
parâmetro estudado, exceto quanto a duas variáveis em dois meridianos nos \\
olhos direitos (OD), e duas variáveis em um meridiano nos olhos esquerdos \\
(OE). Mesmo estas diferenças mostraram-se clinicamente não significantes, \\
por sereminferiores a 0,006mm. Os dois novos parâmetros testados também \\
apresentaram boa reprodutibilidade intra-observador. Conclusão: Este es- \\
tudo confirmou a boa reprodutibilidade intra-observador das variáveis que \\
caracterizam a morfometria do segmento anterior pela UBM.
\end{tabular}

Descritores: Segmento anterior do olho; Ultra-sonografia/instrumentação; Microscopia de video/instrumentação; Valores de referência; Reprodutibilidade de resultados; Transdutores

\section{INTRODUÇ̃̃̃O}

A biomicroscopia ultra-sônica (UBM) permite o estudo das estruturas do segmento anterior através de imagens de alta resolução ${ }^{(1-2)}$. Trata-se de um exame de grande valia, pois permite um estudo microscópico do olho sem o comprometimento da sua integridade. Os mecanismos de algumas patologias do segmento anterior também podem ser mais bem estudados com este exame.

Para reconhecer e classificar as alterações, é fundamental o estabelecimento de um padrão normal. A relevância clínica das medidas obtidas através da UBM depende da reprodutibilidade das mesmas. Podemos destacar na literatura alguns trabalhos que procuraram avaliar a reprodutibilidade intra- e interobservador das medidas em imagens de biomicroscopia ultra-sônica $^{(3-6)}$. Em 1994, foi publicado o primeiro estudo no qual as reprodutibilidades intra- e interobservador foram avaliadas em imagens de UBM 
de quatro pacientes por três observadores distintos em cinco ocasiões diferentes ${ }^{(3)}$. Foi constatada boa reprodutibilidade intra-observador enquanto que a reprodutibilidade interobservador foi pequena. Posteriormente, dois outros estudos analisaram a reprodutibilidade de medidas morfométricas de 50 imagens de UBM realizadas por três observadores em duas ocasiões diferentes ${ }^{(4-5)}$. Foi observada boa reprodutibilidade intra-observador para as medidas que envolviam estruturas bem definidas nas imagens. A reprodutibilidade interobservador foi pequena assim como no trabalho citado anteriormente. Em outro estudo ${ }^{(6)}$, foram avaliados 42 olhos de indivíduos normais utilizados como grupo controle de 54 pacientes portadores de glaucoma primário de ângulo fechado (GPAF). Mesmo não sendo o objetivo do trabalho, os autores avaliaram a reprodutibilidade das medidas encontrando resultados semelhantes aos já descritos.

Não há na literatura relatos de um estudo sistematizado da reprodutibilidade das medidas em um grande número de indivíduos normais. Portanto, o presente trabalho tem como objetivo estudar as variáveis que caracterizam a morfometria do segmento anterior em pacientes com exame oftalmológico normal, em amostra de tamanho significativo, de forma sistematizada, com o intuito de avaliar sua reprodutibilidade intraobservador. Além disso, esse trabalho tem também por objetivo estudar a reprodutibilidade intra-observador de dois outros parâmetros aqui propostos.

\section{MÉTODOS}

Os pacientes deste estudo foram selecionados no Ambulatório do Hospital São Geraldo, Hospital das Clínicas da UFMG, Belo Horizonte, Minas Gerais, no período de maio a dezembro de 1999.

Foram incluídos pacientes voluntários de ambos os sexos, que assinaram o termo de consentimento e que atenderam aos seguintes critérios: 1) idade igual ou superior a 20 anos; 2) exame oftalmológico normal; 3) acuidade visual corrigida melhor ou igual a 0,8 . Foram excluídos do estudo os pacientes que apresentaram: 1) ametropia maior do que 2,00 dioptrias esféricas (de miopia ou de hipermetropia) e/ou 2,00 dioptrias cilíndricas; 2) ceratometria menor do que 41,00 dioptrias ou maior do que 46,00 dioptrias; 3 ) comprimento axial do olho (medido pela ecobiometria) menor do que $22,00 \mathrm{~mm}$ ou maior do que $24,50 \mathrm{~mm}$; 4) história clínica de cirurgias oculares prévias, laserterapia, uso ocular ou sistêmico de antiinflamatórios não esteróides, corticosteróides ou imunossupressores por período superior a duas semanas ou nos últimos dois meses antes do exame oftalmológico; 5) doenças intra-oculares ativas ou cicatrizadas; 6) doenças oculares crônicas ou sistêmicas potencialmente capazes de determinar um comprometimento ocular, excetuando-se hipertensão arterial sistêmica bem controlada; 7) opacidades cristalinianas que determinassem acuidade visual menor do que 0,8 e 8 ) trauma ocular ou cefálico prévio.
A UBM foi realizada empregando-se a versão comercial do biomicroscópio ultra-sônico (Humphrey-Zeiss modelo 840, San Leandro, Califórnia) instalado no Serviço de UBM do Hospital São Geraldo/HC-UFMG. O aparelho possui um transdutor que emite um feixe ultra-sônico linear de $50 \mathrm{MHz}$, tendo uma resolução lateral máxima de 50 micrômetros $(\mu \mathrm{m})$ e uma profundidade de penetração tecidual máxima de $5,0 \mathrm{~mm}$. A resolução do pixel no monitor de imagens é de 11-12 $\mu \mathrm{m}$ na horizontal e de 23-24 $\mu \mathrm{m}$ na vertical.

Os pacientes foram examinados com uma diferença não inferior a sete dias e não superior a quatro semanas em relação ao exame oftalmológico. A UBM foi realizada conforme a técnica previamente descrita na literatura ${ }^{(1-2)}$. A fixação do olhar e a acomodação foram controladas pedindo-se ao paciente que fixasse um ponto no teto da sala de exame com o olho contralateral, e a iluminação da sala de exame foi mantida controlada e constante em 50 lux. Foram obtidas 13 imagens de cada olho estudado, compreendendo uma imagem central (ou axial, para a medida da profundidade da câmara anterior) e três imagens em cortes radiais para cada um dos quatro meridianos pesquisados (superior, nasal, inferior e temporal), de acordo com o posicionamento mais anterior (para as imagens do seio camerular e íris) ou mais posterior (para as imagens da parede escleral e do corpo ciliar) da sonda em relação ao limbo.

Após a obtenção das imagens, foram feitas as medidas consideradas de importância no estudo da morfometria do segmento anterior do olho. Foram feitas duas medidas em cada imagem, pelo mesmo examinador e em dias diferentes com intervalo mínimo de quatro semanas (M1 e M2).

Foram feitas as seguintes medidas, conforme técnica descrita na literatura $^{(2)}$ : a) profundidade da câmara anterior (PCA); b) distância de contato iridolenticular (ILCD); c) distância de abertura do ângulo a 250 e a 500 micrômetros anterior ao esporão escleral (AOD 250 e AOD 500); d) distância da faixa trabecular ao processo ciliar (TCPD); e) espessura iriana (ID1); f) distância írido-zonular (IZD).

Além daquelas, foram estudadas a espessura escleral a 2,0 e a 3,0 $\mathrm{mm}$ posterior ao esporão escleral (EE2 e EE3): medida tomada em um ponto na superfície interna da esclera, localizado respectivamente a 2,0 e a $3,0 \mathrm{~mm}$ posteriormente ao esporão escleral, em uma perpendicular ao plano da esclera (Figura 1); e a espessura do corpo ciliar a 2,0 e a 3,0 $\mathrm{mm}$ posterior ao esporão escleral (ECC2 e ECC3): medida da espessura do corpo ciliar tomada no prolongamento das perpendiculares utilizadas para EE2 e EE3 (Figura 1).

Para a análise estatística da reprodutibilidade intra-observador foi utilizado o teste " $t$ " de Student para amostras pareadas considerando como significativo um valor de $\mathrm{P}<0,05$.

\section{RESULTADOS}

Foram inicialmente selecionados para este estudo 101 pacientes, dos quais $89(88,1 \%)$ tiveram ambos os olhos incluídos e $12(11,9 \%)$ tiveram apenas um dos olhos incluídos. No 


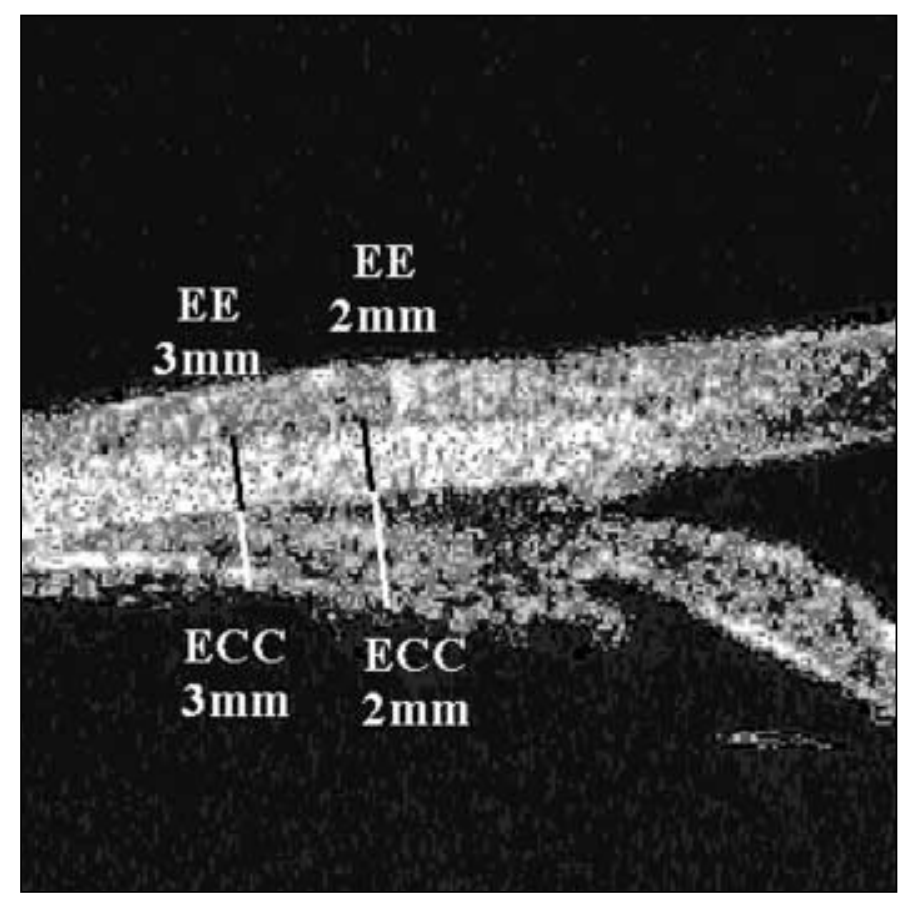

Figura 1 - Representação dos métodos de medida das variáveis espessura escleral (EE) e espessura do corpo ciliar (ECC) a 2 e a $3 \mathrm{~mm}$ do esporão escleral

total, foram estudados 190 olhos, sendo 95 olhos direitos (OD) e 95 olhos esquerdos (OE).

Do total de 101 pacientes incluídos neste estudo, 50 $(49,5 \%)$ pertenciam ao sexo feminino e $51(50,5 \%)$ pertenciam ao sexo masculino. Quanto à raça, observou-se que $44(43,6 \%)$ pacientes eram leucodérmicos, $43(42,6 \%)$ eram feodérmicos e $14(13,8 \%)$ eram melanodérmicos.

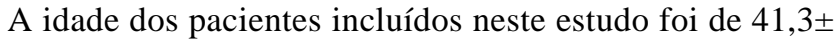
13,9 anos (média \pm desvio-padrão) variando de 20 a 79 anos. $\mathrm{Na}$ distribuição dos pacientes por faixa etária, observou-se que $46(45,6 \%)$ pacientes ( 86 olhos) apresentavam idade entre 20 e 40 anos e $55(54,4 \%)$ pacientes (104 olhos) apresentavam idade acima de 40 anos.

Os dados do exame oftalmológico e de ecobiometria que antecederam a UBM estão resumidos na tabela 1 . Foi observado que os olhos incluídos neste estudo apresentaram pressão intra-ocular dinâmica (média \pm desvio-padrão) de 12,77 $\pm 1,74$ $\mathrm{mmHg}$ (variando de 9 a $17 \mathrm{mmHg}$ ), equivalente-esférico médio de 0,36 $\pm 0,79$ dioptrias (variando de $-2,00$ a 2,00 dioptrias), ceratometria média (KM) de 43,61 $\pm 1,29$ dioptrias (variando de 41,00 a 46,00 dioptrias), e comprimento axial médio de 23,11士 0,63 mm (variando de 22,04 a 24,49 mm). Não foram observadas diferenças estatisticamente significativas para as variáveis dos critérios de inclusão entre olhos direitos e esquerdos ( $\mathrm{p}=0,05$, “ $\mathrm{t}$ ” de Student).

As médias das medidas M1 e M2 de cada parâmetro estudado bem como a diferença entre M1 e M2 segundo o meridiano e o olho analisados encontram-se discriminadas na tabela 2. Para PCA, foi encontrada uma diferença entre M1 e M2

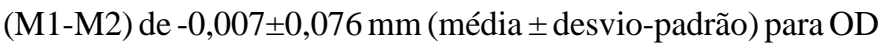
$(P=0,340)$ e de $0,004 \pm 0,026 \mathrm{~mm}$ para $\mathrm{OE}$, diferenças estas não significativas $(P=0,114)$.

Para ILCD, as maiores diferenças observadas entre M1 e M2 foram de $-0,006 \pm 0,050 \mathrm{~mm}$ no meridiano nasal $(\mathrm{N})(P=0,243)$ em OD e de $0,003 \pm 0,032 \mathrm{~mm}$ no meridiano superior em OE, nãosignificantes do ponto de vista estatístico. Nos demais meridianos, as diferenças foram ainda menores e também não significativas.

Para AOD 250, a maior diferença observada entre M1 e M2 ocorreu no meridiano temporal $(0,002 \pm 0,019 \mathrm{~mm} ; P=0,210)$ para OD e nos meridianos nasal e inferior para OE $(0,001 \pm 0,009 \mathrm{~mm}$; $P=0,898$ e $P=0,358$ respectivamente), diferenças estas não significativas, fato também observado nos demais meridianos.

Para AOD 500, a maior diferença entre M1 e M2 ocorreu no meridiano nasal em $\mathrm{OD}(0,007 \pm 0,036 \mathrm{~mm} ; P=0,058)$ e nos meridianos nasal $(0,004 \pm 0,043 \mathrm{~mm} ; P=0,248)$ e inferior $(-0,004 \pm$ $0,043 \mathrm{~mm} ; P=0,396)$ em OE. Estas diferenças e aquelas observadas nos demais meridianos não foram significativas.

Para TCPD, a maior diferença foi encontrada no meridiano temporal $(0,010 \pm 0,065 ; P=0,151)$ em OD e no meridiano superior $(0,007 \pm 0,063 ; P=0,272)$ em OE. Estas diferenças e as dos outros meridianos não foram significativas.

Para ID1, a maior diferença ocorreu nos meridianos inferior $(0,002 \pm 0,019 \mathrm{~mm} ; P=0,387)$ e temporal $(0,002 \pm 0,015 ; P=0,189)$ em OD e no meridiano superior $(0,028 \pm 0,281 ; P=0,336)$ em OE. Estas diferenças não foram significativas assim como nos demais meridianos.

Para IZD, a maior diferença foi observada no meridiano inferior $(0,009 \pm 0,064 ; P=0,160)$ para OD e no meridiano temporal $(0,006 \pm 0,036 ; P=0,339)$ para $\mathrm{OE}$. Estas diferenças não foram significativas. Não obstante, no meridiano superior de OD houve diferença significativa entre M1 e M2 (-0,003 $\pm 0,013$; $P=0,041)$. Nos demais meridianos, as diferenças não foram significativas.

Para EE2, as maiores diferenças em OD ocorreram nos meridianos nasal $(0,003 \pm 0,025 \mathrm{~mm} ; P=0,215)$ e temporal $(0,003$ $\pm 0,027 \mathrm{~mm} ; P=0,254)$ enquanto que em $\mathrm{OE}$ a maior diferença deu-se no meridiano superior $(-0,002 \pm 0,029 \mathrm{~mm} ; P=0,473)$. Já para EE3, a maior diferença em OD ocorreu nos meridianos nasal $(0,006 \pm 0,032 \mathrm{~mm} ; P=0,096)$ enquanto que em $\mathrm{OE}$ as maiores diferenças deram-se nos meridianos nasal e inferior (em ambos: 0,006 $\pm 0,024 \mathrm{~mm} ; P=0,204$ e 0,020 respectivamente). Logo, no meridiano inferior de OE, para EE3 houve diferença estatisticamente significativa entre M1 e M2. Para as demais medidas de EE3 e para todas as medidas de EE2, não houve diferenças significativas.

Para ECC2, a maior diferença encontrada em OD ocorreu no meridiano temporal $(-0,002 \pm 0,028 \mathrm{~mm} ; P=0,513)$ enquanto que no $\mathrm{OE}$ as maiores diferenças ocorreram nos meridianos nasal $(-0,006 \pm 0,028 \mathrm{~mm} ; P=0,545)$ e inferior $(0,006 \pm 0,028 \mathrm{~mm}$; $P=0,033)$. Esta última diferença foi, portanto, estatisticamente significante. Já para ECC3, tanto em OD como em OE as diferenças entre M1 e M2 não ultrapassaram a 0,001mm e não foram significativas exceto no meridiano nasal de OD (M1M2 $=-0,001 \pm 0,004 ; P=0,015)$. 


\begin{tabular}{|c|c|c|c|c|c|c|}
\hline \multirow[b]{2}{*}{ Variável } & \multirow[b]{2}{*}{ Olho } & \multicolumn{4}{|c|}{ Medidas descritivas } & \multirow[b]{2}{*}{$\mathbf{p}$} \\
\hline & & Mínimo & Máximo & Média & Desvio-padrão & \\
\hline Po & OD & 9,00 & 16,00 & 12,64 & 1,72 & \multirow[t]{3}{*}{0,200} \\
\hline \multirow{2}{*}{$\begin{array}{l}\text { Dinâmica } \\
(\mathrm{mmHg})\end{array}$} & OE & 10,00 & 17,00 & 12,91 & 1,77 & \\
\hline & Geral & 9,00 & 17,00 & 12,77 & 1,74 & \\
\hline Po & OD & 9,00 & 18,00 & 12,65 & 2,17 & \multirow[t]{3}{*}{0,470} \\
\hline \multirow{2}{*}{$\begin{array}{l}\text { Sob ciclo } \\
\text { (mmHg) }\end{array}$} & OE & 9,00 & 18,00 & 12,71 & 2,13 & \\
\hline & Geral & 9,00 & 18,00 & 12,68 & 2,14 & \\
\hline EqEsf & OD & $-2,00$ & 2,00 & 0,41 & 0,76 & \multirow[t]{3}{*}{0,370} \\
\hline \multirow[t]{2}{*}{ (Dioptrias) } & OE & $-2,00$ & 2,00 & 0,31 & 0,82 & \\
\hline & Geral & $-2,00$ & 2,00 & 0,36 & 0,79 & \\
\hline KM & OD & 41,00 & 46,00 & 43,61 & 1,31 & \multirow[t]{3}{*}{0,409} \\
\hline \multirow[t]{2}{*}{ (Dioptrias) } & OE & 41,25 & 46,00 & 43,61 & 1,27 & \\
\hline & Geral & 41,00 & 46,00 & 43,61 & 1,29 & \\
\hline CAx & OD & 22,04 & 24,49 & 23,12 & 0,62 & \multirow[t]{3}{*}{0,200} \\
\hline \multirow[t]{2}{*}{$(\mathrm{mm})$} & OE & 22,10 & 24,49 & 23,09 & 0,63 & \\
\hline & Geral & 22,04 & 24,49 & 23,11 & 0,63 & \\
\hline $\mathrm{CA}$ & OD & 2,56 & 3,90 & 3,21 & 0,28 & \multirow[t]{3}{*}{0,669} \\
\hline \multirow[t]{2}{*}{$(\mathrm{mm})$} & OE & 2,61 & 3,84 & 3,21 & 0,30 & \\
\hline & Geral & 2,56 & 3,90 & 3,21 & 0,29 & \\
\hline Cristalino & OD & 3,24 & 5,17 & 4,10 & 0,35 & \multirow[t]{3}{*}{0,480} \\
\hline$(\mathrm{EL})$ & OE & 3,39 & 5,15 & 4,13 & 0,37 & \\
\hline$(\mathrm{mm})$ & Geral & 3,24 & 5,17 & 4,11 & 0,36 & \\
\hline
\end{tabular}

Em resumo, no olho direito não foram observadas diferenças estatisticamente significativas entre M1 e M2 para as variáveis estudadas exceto para IZD no meridiano superior (M1 - M2 $=-0,003 \pm 0,013 \mathrm{~mm} ; P=0,041$, tabela 2$)$, e para ECC3 no meridiano nasal (M1 - M2 $=-0,001 \pm 0,004 \mathrm{~mm}, P=0,015$, tabela 2). No olho esquerdo, não se observaram diferenças estatisticamente significativas entre M1 e M2 para as variáveis estudadas exceto para EE3 (M1 - M2 $=0,006 \pm 0,024 \mathrm{~mm}$, $P=0,02$, tabela 2) e ECC2 (M1 - M2 $=0,006 \pm 0,028 \mathrm{~mm}, P=0,033$, tabela 2), ambas no meridiano inferior.

\section{DISCUSSÃO}

Este é o primeiro estudo a ser publicado na literatura nacional a avaliar sistematicamente a reprodutibilidade intra-observador das medidas morfométricas obtidas por meio da UBM. A determinação da reprodutibilidade das medidas é passo preliminar fundamental que deve anteceder qualquer estudo quantitativo empregando-se exames de imagem ${ }^{(3)}$. A reprodutibilidade consiste na determinação do grau de proximidade que duas ou mais séries de observações de uma mesma variável apresentam entre si, podendo ser afetada por vários fatores, como diferenças sistemáticas na mensuração quando feitas por um mesmo observador, por observadores diferentes ou pelo instrumento de aferição utilizado; pode ainda derivar de variações fisiológicas do parâmetro investigado ${ }^{(3-4)}$. No presente estudo, a determinação da reprodutibilidade teve por objetivo quantificar o erro intra-observador. Para este fim, determinou-se o erro de medida causado pela interpretação da mesma imagem pelo observador em dois momentos diferentes. Portanto, os resultados observados não sofreram a influência dos outros fatores externos que interferem na medida da reprodutibilidade intra-observador ${ }^{(3)}$.

Eliminados esses fatores externos de variabilidade, a reprodutibilidade intra-observador das medidas feitas em imagens de UBM depende do erro ou variabilidade de mensuração relacionado à interpretação humana das imagens ${ }^{(3)}$. Essa variabilidade está, por sua vez, relacionada à clareza ou facilidade de identificar os marcos anatômicos que definem certos parâmetros e a variações na qualidade das imagens $\mathbf{s}^{(4)}$. Parâmetros morfométricos definidos por marcos anatômicos facilmente discerníveis apresentam menor probabilidade de erro de interpretação por parte do examinador e portanto possuem boa reprodutibilidade. Entre esses parâmetros, estão as variáveis PCA e a medida da espessura escleral (EE). Parâmetros morfométricos que envolvem a íris e a abertura da câmara anterior apresentam maior susceptibilidade de erro de interpretação pelo examinador, por dificuldades relacionadas ao estabelecimento dos marcos anatômicos das medidas. A maior ou menor facilidade de mensuração pela UBM também está relacionada à qualidade das imagens selecionadas, que influencia igualmente a clareza com que os marcos anatômicos podem ser encontrados. A boa qualidade das imagens pode 


\begin{tabular}{|c|c|c|c|c|c|c|c|}
\hline & & & M1 (mm) & M2 (mm) & M1 - M2 (mm) & & \\
\hline Variável & Olho & Meridiano & Média \pm desvio padrão & Média \pm desvio padrão & Média \pm desvio padrão & $P$ & Conclusão \\
\hline \multirow[t]{2}{*}{ PCA } & OD & & $2,824 \pm 0,276$ & $2,831 \pm 0,279$ & $-0,007 \pm 0,076$ & 0,340 & $\mathrm{M} 1$ = M2 \\
\hline & $\mathrm{OE}$ & & $2,840 \pm 0,278$ & $2,835 \pm 0,277$ & $0,004 \pm 0,026$ & 0,114 & $\mathrm{M} 1=\mathrm{M} 2$ \\
\hline \multirow[t]{8}{*}{ ILCD } & OD & Superior & $0,304 \pm 0,121$ & $0,304 \pm 0,120$ & $0,001 \pm 0,004$ & 0,087 & $\mathrm{M} 1=\mathrm{M} 2$ \\
\hline & & Nasal & $0,347 \pm 0,135$ & $0,353 \pm 0,144$ & $-0,006 \pm 0,050$ & 0,243 & $\mathrm{M} 1=\mathrm{M} 2$ \\
\hline & & Inferior & $0,353 \pm 0,136$ & $0,351 \pm 0,130$ & $0,002 \pm 0,070$ & 0,801 & $\mathrm{M} 1=\mathrm{M} 2$ \\
\hline & & Temporal & $0,331 \pm 0,143$ & $0,325 \pm 0,132$ & $0,005 \pm 0,043$ & 0,220 & $\mathrm{M} 1=\mathrm{M} 2$ \\
\hline & $\mathrm{OE}$ & Superior & $0,336 \pm 0,126$ & $0,332 \pm 0,121$ & $0,003 \pm 0,032$ & 0,347 & $\mathrm{M} 1=\mathrm{M} 2$ \\
\hline & & Nasal & $0,380 \pm 0,145$ & $0,380 \pm 0,144$ & $0,001 \pm 0,009$ & 0,637 & $\mathrm{M} 1=\mathrm{M} 2$ \\
\hline & & Inferior & $0,359 \pm 0,124$ & $0,358 \pm 0,124$ & $0,001 \pm 0,009$ & 0,248 & $\mathrm{M} 1=\mathrm{M} 2$ \\
\hline & & Temporal & $0,318 \pm 0,128$ & $0,317 \pm 0,127$ & $0,001 \pm 0,007$ & 0,126 & $\mathrm{M} 1=\mathrm{M} 2$ \\
\hline$A O D$ & OD & Superior & $0,204 \pm 0,084$ & $0,204 \pm 0,083$ & $0,001 \pm 0,004$ & 0,177 & $\mathrm{M} 1=\mathrm{M} 2$ \\
\hline \multirow[t]{7}{*}{250} & & Nasal & $0,258 \pm 0,109$ & $0,258 \pm 0,108$ & $<0,001 \pm 0,018$ & 0,960 & $\mathrm{M} 1=\mathrm{M} 2$ \\
\hline & & Inferior & $0,227 \pm 0,089$ & $0,228 \pm 0,091$ & $-0,001 \pm 0,021$ & 0,599 & $\mathrm{M} 1=\mathrm{M} 2$ \\
\hline & & Temporal & $0,278 \pm 0,128$ & $0,275 \pm 0,123$ & $0,002 \pm 0,019$ & 0,210 & $\mathrm{M} 1=\mathrm{M} 2$ \\
\hline & OE & Superior & $0,206 \pm 0,076$ & $0,206 \pm 0,075$ & $<0,001 \pm 0,004$ & 0,543 & $\mathrm{M} 1=\mathrm{M} 2$ \\
\hline & & Nasal & $0,251 \pm 0,108$ & $0,250 \pm 0,105$ & $0,001 \pm 0,009$ & 0,898 & $\mathrm{M} 1=\mathrm{M} 2$ \\
\hline & & Inferior & $0,243 \pm 0,101$ & $0,241 \pm 0,100$ & $0,001 \pm 0,009$ & 0,358 & $\mathrm{M} 1=\mathrm{M} 2$ \\
\hline & & Temporal & $0,260 \pm 0,102$ & $0,259 \pm 0,102$ & $<0,001 \pm 0,012$ & 0,804 & $\mathrm{M} 1=\mathrm{M} 2$ \\
\hline AOD & OD & Superior & $0,311 \pm 0,140$ & $0,305 \pm 0,126$ & $0,006 \pm 0,049$ & 0,213 & $\mathrm{M} 1=\mathrm{M} 2$ \\
\hline \multirow[t]{7}{*}{500} & & Nasal & $0,382 \pm 0,155$ & $0,375 \pm 0,154$ & $0,007 \pm 0,036$ & 0,058 & $\mathrm{M} 1=\mathrm{M} 2$ \\
\hline & & Inferior & $0,345 \pm 0,130$ & $0,348 \pm 0,133$ & $-0,002 \pm 0,018$ & 0,216 & $\mathrm{M} 1=\mathrm{M} 2$ \\
\hline & & Temporal & $0,428 \pm 0,205$ & $0,422 \pm 0,203$ & $0,006 \pm 0,034$ & 0,092 & $\mathrm{M} 1=\mathrm{M} 2$ \\
\hline & OE & Superior & $0,317 \pm 0,152$ & $0,316 \pm 0,153$ & $0,001 \pm 0,009$ & 0,293 & $\mathrm{M} 1=\mathrm{M} 2$ \\
\hline & & Nasal & $0,375 \pm 0,160$ & $0,371 \pm 0,155$ & $0,004 \pm 0,043$ & 0,248 & $\mathrm{M} 1=\mathrm{M} 2$ \\
\hline & & Inferior & $0,351 \pm 0,138$ & $0,355 \pm 0,149$ & $-0,004 \pm 0,043$ & 0,396 & $\mathrm{M} 1=\mathrm{M} 2$ \\
\hline & & Temporal & $0,386 \pm 0,144$ & $0,384 \pm 0,146$ & $0,002 \pm 0,028$ & 0,572 & $\mathrm{M} 1=\mathrm{M} 2$ \\
\hline \multirow[t]{8}{*}{ TCPD } & OD & Superior & $0,877 \pm 0,174$ & $0,879 \pm 0,174$ & $-0,001 \pm 0,012$ & 0,473 & $\mathrm{M} 1=\mathrm{M} 2$ \\
\hline & & Nasal & $0,912 \pm 0,170$ & $0,910 \pm 0,167$ & $0,001 \pm 0,024$ & 0,556 & $\mathrm{M} 1=\mathrm{M} 2$ \\
\hline & & Inferior & $0,885 \pm 0,162$ & $0,889 \pm 0,161$ & $-0,003 \pm 0,031$ & 0,307 & $\mathrm{M} 1=\mathrm{M} 2$ \\
\hline & & Temporal & $0,897 \pm 0,179$ & $0,888 \pm 0,188$ & $0,010 \pm 0,065$ & 0,151 & $\mathrm{M} 1=\mathrm{M} 2$ \\
\hline & OE & Superior & $0,882 \pm 0,185$ & $0,875 \pm 0,194$ & $0,007 \pm 0,063$ & 0,272 & $\mathrm{M} 1=\mathrm{M} 2$ \\
\hline & & Nasal & $0,902 \pm 0,161$ & $0,906 \pm 0,155$ & $0,004 \pm 0,043$ & 0,248 & $\mathrm{M} 1=\mathrm{M} 2$ \\
\hline & & Inferior & $0,917 \pm 0,169$ & $0,913 \pm 0,171$ & $0,005 \pm 0,040$ & 0,269 & $\mathrm{M} 1=\mathrm{M} 2$ \\
\hline & & Temporal & $0,884 \pm 0,157$ & $0,878 \pm 0,168$ & $0,006 \pm 0,063$ & 0,341 & $\mathrm{M} 1=\mathrm{M} 2$ \\
\hline \multirow[t]{8}{*}{ ID1 } & OD & Superior & $0,338 \pm 0,063$ & $0,337 \pm 0,062$ & $0,001 \pm 0,009$ & 0,275 & $\mathrm{M} 1=\mathrm{M} 2$ \\
\hline & & Nasal & $0,353 \pm 0,068$ & $0,352 \pm 0,070$ & $<0,001 \pm 0,012$ & 0,924 & $\mathrm{M} 1=\mathrm{M} 2$ \\
\hline & & Inferior & $0,353 \pm 0,072$ & $0,351 \pm 0,070$ & $0,002 \pm 0,019$ & 0,387 & $\mathrm{M} 1=\mathrm{M} 2$ \\
\hline & & Temporal & $0,341 \pm 0,064$ & $0,339 \pm 0,064$ & $0,002 \pm 0,015$ & 0,189 & $\mathrm{M} 1=\mathrm{M} 2$ \\
\hline & OE & Superior & $0,378 \pm 0,289$ & $0,350 \pm 0,084$ & $0,028 \pm 0,281$ & 0,336 & $\mathrm{M} 1=\mathrm{M} 2$ \\
\hline & & Nasal & $0,347 \pm 0,071$ & $0,347 \pm 0,072$ & $0,001 \pm 0,014$ & 0,987 & $\mathrm{M} 1=\mathrm{M} 2$ \\
\hline & & Inferior & $0,361 \pm 0,066$ & $0,360 \pm 0,067$ & $0,001 \pm 0,014$ & 0,441 & $\mathrm{M} 1=\mathrm{M} 2$ \\
\hline & & Temporal & $0,337 \pm 0,074$ & $0,341 \pm 0,067$ & $-0,004 \pm 0,047$ & 0,395 & $\mathrm{M} 1=\mathrm{M} 2$ \\
\hline \multirow[t]{8}{*}{ IZD } & OD & Superior & $0,626 \pm 0,100$ & $0,629 \pm 0,100$ & $-0,003 \pm 0,013$ & 0,041 & M1 < M2 \\
\hline & & Nasal & $0,583 \pm 0,088$ & $0,584 \pm 0,090$ & $-0,001 \pm 0,036$ & 0,852 & $\mathrm{M} 1=\mathrm{M} 2$ \\
\hline & & Inferior & $0,598 \pm 0,090$ & $0,587 \pm 0,097$ & $0,009 \pm 0,064$ & 0,160 & $\mathrm{M} 1=\mathrm{M} 2$ \\
\hline & & Temporal & $0,589 \pm 0,096$ & $0,589 \pm 0,095$ & $-0,001 \pm 0,024$ & 0,698 & $\mathrm{M} 1=\mathrm{M} 2$ \\
\hline & OE & Superior & $0,647 \pm 0,110$ & $0,641 \pm 0,109$ & $0,005 \pm 0,033$ & 0,157 & $\mathrm{M} 1=\mathrm{M} 2$ \\
\hline & & Nasal & $0,592 \pm 0,103$ & $0,594 \pm 0,106$ & $-0,005 \pm 0,026$ & 0,644 & $\mathrm{M} 1=\mathrm{M} 2$ \\
\hline & & Inferior & $0,607 \pm 0,088$ & $0,601 \pm 0,089$ & $0,005 \pm 0,026$ & 0,087 & $\mathrm{M} 1=\mathrm{M} 2$ \\
\hline & & Temporal & $0,620 \pm 0,113$ & $0,614 \pm 0,115$ & $0,006 \pm 0,036$ & 0,339 & $\mathrm{M} 1=\mathrm{M} 2$ \\
\hline \multirow[t]{9}{*}{ EE2 } & $O D$ & Superior & $0,479 \pm 0,048$ & $0,480 \pm 0,049$ & $-0,001 \pm 0,012$ & 0,464 & $\mathrm{M} 1=\mathrm{M} 2$ \\
\hline & & Nasal & $0,518 \pm 0,054$ & $0,515 \pm 0,058$ & $0,003 \pm 0,025$ & 0,215 & $\mathrm{M} 1=\mathrm{M} 2$ \\
\hline & & Inferior & $0,530 \pm 0,054$ & $0,530 \pm 0,045$ & $<0,001 \pm 0,032$ & 0,918 & $\mathrm{M} 1=\mathrm{M} 2$ \\
\hline & & Temporal & $0,516 \pm 0,047$ & $0,513 \pm 0,046$ & $0,003 \pm 0,027$ & 0,254 & $\mathrm{M} 1=\mathrm{M} 2$ \\
\hline & $\mathrm{OE}$ & Superior & $0,486 \pm 0,049$ & $0,489 \pm 0,059$ & $-0,002 \pm 0,029$ & 0,473 & $\mathrm{M} 1=\mathrm{M} 2$ \\
\hline & & Nasal & $0,510 \pm 0,046$ & $0,510 \pm 0,047$ & $0,001 \pm 0,008$ & 0,826 & $\mathrm{M} 1=\mathrm{M} 2$ \\
\hline & & Inferior & $0,534 \pm 0,045$ & $0,533 \pm 0,046$ & $0,001 \pm 0,008$ & 0,228 & $\mathrm{M} 1=\mathrm{M} 2$ \\
\hline & & Temporal & $0,524 \pm 0,052$ & $0,524 \pm 0,052$ & $0,001 \pm 0,008$ & 0,400 & $\mathrm{M} 1=\mathrm{M} 2$ \\
\hline & & & & & & & (Cont.) \\
\hline
\end{tabular}




\begin{tabular}{|c|c|c|c|c|c|c|c|}
\hline & & & M1 (mm) & M2 (mm) & M1 - M2 (mm) & & \\
\hline Variável & Olho & Meridiano & Média \pm desvio padrão & Média \pm desvio padrão & Média \pm desvio padrão & $\boldsymbol{P}$ & Conclusão \\
\hline \multirow[t]{8}{*}{ EE3 } & OD & Superior & $0,445 \pm 0,041$ & $0,444 \pm 0,041$ & $0,001 \pm 0,005$ & 0,217 & $\mathrm{M} 1=\mathrm{M} 2$ \\
\hline & & Nasal & $0,488 \pm 0,057$ & $0,482 \pm 0,066$ & $0,006 \pm 0,032$ & 0,096 & $\mathrm{M} 1=\mathrm{M} 2$ \\
\hline & & Inferior & $0,501 \pm 0,051$ & $0,500 \pm 0,051$ & $0,001 \pm 0,007$ & 0,073 & $M 1=M 2$ \\
\hline & & Temporal & $0,483 \pm 0,058$ & $0,486 \pm 0,050$ & $-0,003 \pm 0,031$ & 0,436 & $\mathrm{M} 1=\mathrm{M} 2$ \\
\hline & OE & Superior & $0,451 \pm 0,048$ & $0,451 \pm 0,049$ & $<0,001 \pm 0,004$ & 0,581 & $\mathrm{M} 1=\mathrm{M} 2$ \\
\hline & & Nasal & $0,483 \pm 0,052$ & $0,486 \pm 0,055$ & $-0,006 \pm 0,024$ & 0,204 & $\mathrm{M} 1=\mathrm{M} 2$ \\
\hline & & Inferior & $0,503 \pm 0,050$ & $0,497 \pm 0,056$ & $0,006 \pm 0,024$ & 0,020 & $M 1>M 2$ \\
\hline & & Temporal & $0,485 \pm 0,079$ & $0,486 \pm 0,072$ & $-0,001 \pm 0,033$ & 0,675 & $\mathrm{M} 1=\mathrm{M} 2$ \\
\hline \multirow[t]{8}{*}{ ECC2 } & OD & Superior & $0,306 \pm 0,101$ & $0,306 \pm 0,099$ & $<0,001 \pm 0,010$ & 0,780 & $M 1=M 2$ \\
\hline & & Nasal & $0,325 \pm 0,087$ & $0,325 \pm 0,087$ & $<0,001 \pm 0,009$ & 0,847 & $M 1=M 2$ \\
\hline & & Inferior & $0,342 \pm 0,085$ & $0,340 \pm 0,084$ & $0,001 \pm 0,013$ & 0,285 & $\mathrm{M} 1=\mathrm{M} 2$ \\
\hline & & Temporal & $0,339 \pm 0,083$ & $0,330 \pm 0,087$ & $-0,002 \pm 0,028$ & 0,513 & $M 1=M 2$ \\
\hline & OE & Superior & $0,293 \pm 0,089$ & $0,296 \pm 0,085$ & $-0,003 \pm 0,034$ & 0,399 & $M 1=M 2$ \\
\hline & & Nasal & $0,320 \pm 0,074$ & $0,322 \pm 0,079$ & $-0,006 \pm 0,028$ & 0,545 & $M 1=M 2$ \\
\hline & & Inferior & $0,349 \pm 0,088$ & $0,342 \pm 0,086$ & $0,006 \pm 0,028$ & 0,033 & $M 1>M 2$ \\
\hline & & Temporal & $0,344 \pm 0,092$ & $0,343 \pm 0,092$ & $0,001 \pm 0,009$ & 0,508 & $M 1=M 2$ \\
\hline \multirow[t]{8}{*}{ ECC3 } & OD & Superior & $0,192 \pm 0,113$ & $0,193 \pm 0,113$ & $-0,001 \pm 0,008$ & 0,129 & $M 1=M 2$ \\
\hline & & Nasal & $0,201 \pm 0,049$ & $0,203 \pm 0,049$ & $-0,001 \pm 0,004$ & 0,015 & $M 1<M 2$ \\
\hline & & Inferior & $0,226 \pm 0,052$ & $0,225 \pm 0,052$ & $0,001 \pm 0,006$ & 0,372 & $M 1=M 2$ \\
\hline & & Temporal & $0,203 \pm 0,049$ & $0,204 \pm 0,050$ & $<0,001 \pm 0,010$ & 0,728 & $\mathrm{M} 1=\mathrm{M} 2$ \\
\hline & OE & Superior & $0,175 \pm 0,046$ & $0,176 \pm 0,047$ & $-0,001 \pm 0,007$ & 0,109 & $\mathrm{M} 1=\mathrm{M} 2$ \\
\hline & & Nasal & $0,198 \pm 0,049$ & $0,197 \pm 0,046$ & $<0,001 \pm 0,006$ & 0,520 & $\mathrm{M} 1=\mathrm{M} 2$ \\
\hline & & Inferior & $0,226 \pm 0,056$ & $0,226 \pm 0,055$ & $<0,001 \pm 0,006$ & 0,912 & $M 1=M 2$ \\
\hline & & Temporal & $0,208 \pm 0,048$ & $0,207 \pm 0,047$ & $0,001 \pm 0,005$ & 0,403 & $M 1=M 2$ \\
\hline \multicolumn{8}{|c|}{$\begin{array}{l}\text { PCA: Profundidade central da câmara anterior; ILCD: Área de contato írido-lenticular; AOD250: Distância de abertura angular a 250 micra do esporão escleral; AOD500: } \\
\text { Distância de abertura angular a } 500 \text { micra do esporão escleral; TCPD: Distância da faixa trabecular ao processo ciliar; ID1: Espessura iriana; IZD: Distância írido-- } \\
\text { zonular; EE2: Espessura escleral a 2,0 mm posterior ao esporão escleral; EE3: Espessura escleral a 3,0 mm posterior ao esporão escleral; ECC2: Espessura do corpo } \\
\text { ciliar a 2,0 mm posterior ao esporão escleral; ECC3: Espessura do corpo ciliar a 3,0 mm posterior ao esporão escleral; M1: 1a medida efetuada; M2: } 2^{a} \text { medida efetuada; } \\
\text { M1-M2: Diferença entre as medidas; OD: Olho direito; OE: Olho esquerdo. } \\
\text { Nota: A probabilidade de significância refere-se ao teste t de Student para amostras pareadas. } n=190 \text { olhos. }\end{array}$} \\
\hline
\end{tabular}

ser garantida quando fatores como o posicionamento do paciente, a luminosidade da sala de exame, a fixação do olhar e o controle da acomodação do olho contralateral são devidamente padronizados ${ }^{(4)}$. Além disto, a maior definição do elemento anatômico observado é obtida quando ele situa-se perpendicular ao feixe ultra-sônico, o que é função direta do correto posicionamento da sonda visando o controle da sua rotação e da sua inclinação ${ }^{(4)}$.

Observou-se que, embora diferenças estatisticamente significativas entre as medidas M1 e M2 tenham sido encontradas para duas variáveis em dois meridianos no OD e duas variáveis em um meridiano no OE, em todos estes casos a diferença entre M1 e M2 foi menor do que $6 \mu \mathrm{m}$, por isso sem significado clínico, uma vez que extrapolam o limite de precisão do aparelho, que é de 12 x $24 \mu \mathrm{m}^{(4)}$. Pode-se dizer que, em termos clínicos, M1=M2 para todas as variáveis estudadas. Portanto, elas apresentaram boa reprodutibilidade intra-observador.

Das 11 variáveis medidas neste trabalho, sete apresentam estudos de reprodutibilidade prévios relatados na literatura (PCA, AOD 250, AOD 500, ILCD, TCPD, ID1 e IZD). Quatro outras variáveis aqui estudadas são inéditas quanto à sua reprodutibilidade (EE2, EE3, ECC2 e ECC3). De maneira similar ao presente trabalho, outros estudos também demonstraram uma boa reprodutibilidade intra-observador para os parâmetros PCA, AOD 500, TCPD, ID1 e IZD ${ }^{(3,6)}$. Apenas um trabalho anterior $^{(3)}$ analisou a reprodutibilidade do parâmetro AOD 250, que foi considerado satisfatório, como no presente estudo. $\mathrm{O}$ parâmetro ILCD apresentou boa reprodutibilidade intra-observador confirmando observação prévia na literatura ${ }^{(3)}$ embora outro trabalho tenha encontrado uma reprodutibilidade apenas razoável para esse parâmetro ${ }^{(6)}$.

A reprodutibilidade da espessura escleral (EE) é habitualmente medida por uma perpendicular ao esporão escleral ${ }^{(3-5)}$. Não foram encontrados trabalhos na literatura reportando a reprodutibilidade da $\mathrm{EE}$ a 2 e $3 \mathrm{~mm}$ do esporão escleral nem a reprodutibilidade da espessura do corpo ciliar (ECC) nas mesmas posições. Como demonstrado neste trabalho, a reprodutibilidade desses parâmetros foi considerada satisfatória.

Apenas um estudo ${ }^{(4)}$ procurou medir a reprodutibilidade de alguns parâmetros morfométricos de acordo com o meridiano. Nenhum trabalho prévio na literatura separou a reprodutibilidade de acordo com o olho estudado. Estas avaliações são importantes para se determinar se existe a influência do meridiano e do olho examinado sobre a reprodutibilidade. A influência destes fatores poderia dever-se a dificuldades inerentes à obtenção da imagem. O meridiano temporal é reconhecido como o 
meridiano onde o acesso da sonda de UBM se faz com mais facilidade $^{(1)}$, o que confere melhor perpendicularidade do feixe ultra-sônico à estrutura avaliada e portanto maior clareza dessa estrutura. De maneira similar, examinadores destros poderiam teoricamente ter maior facilidade de posicionar a sonda de UBM para estudos do OD que do OE. Dentre os 11 parâmetros analisados pelo presente estudo, apenas o AOD 500 foi avaliado de acordo com o meridiano estudado ${ }^{(4)}$; não houve influência do meridiano sobre este parâmetro. No presente estudo, todavia, observou-se que as variáveis IZD no meridiano superior e ECC3 no meridiano nasal de OD apresentaram variação estatística assim como EE3 e ECC2 no meridiano inferior de OE. Entretanto, não é possível determinar apenas com os dados analisados se essa variação ocorreu em função da qualidade do parâmetro estudado, do meridiano ou do olho examinado ou por uma combinação desses fatores.

Algumas limitações aplicam-se quando os resultados desse estudo são comparados com os demais da literatura. O objetivo do presente estudo foi identificar se havia boa reprodutibilidade intra-observador dos parâmetros morfométricos medidos pela UBM em duas ocasiões distintas. O propósito deste método é determinar se a média dessas medidas pode ser utilizada para outras comparações morfométricas, como por exemplo, a comparação das variáveis entre meridianos. Por esta razão, a estatística empregada foi o "t"de Student e uma vez que houve boa reprodutibilidade das variáveis estudadas, o estudo demonstrou que a média dessas medidas pode ser utilizada. Os trabalhos prévios $^{(3-6)}$ sobre reprodutibilidade procuraram analisar o grau de dispersão ao redor da média que medidas repetidas de uma variável produzem. Embora alguma diferença de informação possa ocorrer entre as metodologias, ainda assim comparações podem ser feitas.

Finalmente, observa-se que os dados obtidos no exame oftalmológico e nos exames biométricos dos olhos dos pacientes incluídos no presente estudo mostrou-se muito similar àquela encontrada em estudos de olhos normais na literatu$\mathrm{ra}^{(7)}$, comprovando ser a amostra deste estudo composta de olhos normais. Da mesma forma, o presente estudo exibe a maior amostra de olhos normais já estudada quanto à reprodutibilidade das medidas morfométricas obtidas pela UBM na literatura. Estes fatores destacam a relevância estatística deste estudo.

Pode-se concluir que as variáveis estudadas apresentam boa reprodutibilidade intra-observador quando medidas nas imagens de UBM.

\section{ABSTRACT}

Purpose: To systematically study the anterior segment morphometric measurements by ultrasound biomicroscopy (UBM) in a large population of normal eyes, to evaluate their intraobserver reproducibility. Two new measurements are proposed and also tested concerning their intra-observer reproducibility. Methods: One hundred ninety eyes of 101 patients with normal ophthalmic examination were studied by UBM. Anterior chamber depth (ACD) along with 11 different morphometric parameters that characterize the anterior segment morphometry were measured in the superior, nasal, inferior and temporal meridians. All measurements were performed twice with a minimum interval of four weeks between them. Results: There were no statistical significant differences between the first (M1) and second (M2) measurements and regarding each studied parameter except for 2 variables in 2 meridians in the right eyes (OD) and 2 variables of one meridian in the left eyes (OS). Even in these cases, the largest difference between M1 and M2 was less than $0.006 \mathrm{~mm}$, which was not clinically significant. The new tested parameters also presented a good intra-observer reproducibility. Conclusion: This study confirmed the good intraobserver reproducibility of the variables that characterize anterior segment morphometry by UBM.

Keywords: Anterior eye segment; Ultrasonography/instrumentation; Microscopy, Video/instrumentation; Reference values; Reproducibility of results; Transducer

\section{REFERÊNCIAS}

1. Pavlin CJ, Harasiewicz K, Sherar MD, Foster FS. Clinical use of ultrasound biomicroscopy. Ophthalmology. 1991;98(3):287-95.

2. Pavlin CJ, Harasiewicz K, Foster FS. Ultrasound biomicroscopy of anterior segment structures in normal and glaucomatous eyes. Am J Ophthalmol. 1992;113(4):381-9.

3. Tello C, Liebmann J, Potash SD, Cohen H, Ritch R. Measurement of ultrasound biomicroscopy images: intraobserver and interobserver reliability. Invest Ophthalmol Vis Sci. 1994;35(9):3549-52.

4. Urbak SF. Ultrasound biomicroscopy. I. Precision of measurements. Acta Ophthalmol Scand. 1998;76(4):447-55.

5. Urbak SF, Pedersen JK, Thorsen TT. Ultrasound biomicroscopy. II. Intraobserver and interobserver reproducibility of measurements. Acta Ophthalmol Scand. 1998;76(5):546-9.

6. Marchini G, Pagliarusco A, Toscano A, Tosi R, Brunelli C, Bonomi L. Ultrasound biomicroscopic and conventional ultrasonographic study of ocular dimensions in primary angle-closure glaucoma. Ophthalmology. 1998;105(11): 2091-8.

7. Karesh JW. Topographic anatomy of the eye: an overview. In: Tasman W, Jarger EA, editors. Duane's Foundations of Clinical Ophthalmology. Philadelphia: Lippincott Williams \& Wilkins, 1994. p.1-30. 